\title{
The Potential Functional Interaction of Biglycan and WISP-1 in Controlling Differentiation and Proliferation of Osteogenic Cells
}

\author{
Colette A. Inkson Mitsuaki Ono Yanming Bi Sergei A. Kuznetsov \\ Larry W. Fisher Marian F. Young \\ Craniofacial and Skeletal Diseases Branch, National Institute of Dental and Craniofacial Research, \\ National Institutes of Health, DHHS Bethesda, Bethesda, Md., USA
}

\section{Key Words}

Biglycan · WISP-1/CCN4 • WISP-1va • Osteogenesis •

Proliferation

\begin{abstract}
Biglycan (BGN) and WISP-1 are 2 extracellular matrix proteins that bind to each other and colocalize in mineralizing tissue. Here we show that WISP-1 abrogates the repression of proliferation in bone marrow stromal cells induced by BGN. We also demonstrate that WISP-1 and its variant WISP-1va can alleviate the repressed osteogenic differentiation caused by the absence of BGN. These preliminary data suggest that WISP-1 and BGN may functionally interact and control each other's activity, thus regulating the differentiation and proliferation of osteogenic cells. Copyright $\odot 2008$ S. Karger AG, Basel
\end{abstract}

\section{Introduction}

Biglycan (BGN) is a member of the small leucine-rich proteoglycan (SLRP) family that was originally discovered as an abundant component of mineralized tissues [Fisher et al., 1983]. Members of the SLRP family are characterized by their unique core proteins that are composed predominantly of a repeating structural motif rich in leucine. Many of the 13 SLRP members have been detected in mineralizing tissue, but their precise roles are complex and still uncertain. When BGN was depleted by gene targeting, mutant mice developed age-related osteopenia [Xu et al., 1998] due to a decrease in osteogenic progenitor number [Chen et al., 2002] and osteoblastic differentiation from reduced binding of bone morphogenetic protein (BMP)- 4 to osteogenic precursors [Chen et al., 2004]. The importance of BGN in mineralization is supported by studies using BGN-targeted antisense technology in cultured osteoblast-like cells [Parisuthiman et al., 2005] or with in vitro mineralization assays [Boskey et al., 1997]. With this foundation in understanding the role of BGN in mineralization, additional details on how BGN can control these processes and whether other associated factors are involved now need to be resolved.

Wnt-1-induced secreted protein-1 (WISP-1) is a member of the CCN family (named after the founding members CYR61, CTGF and Nov) that has been found to bind to

\section{Abbreviations used in this paper}

\begin{tabular}{ll}
\hline AP & alkaline phosphatase \\
BGN & biglycan \\
BMP & bone morphogenetic protein \\
hBMSCs & human bone marrow stromal cells \\
mBMSCs & mouse bone marrow stromal cells \\
SLRP & small leucine-rich proteoglycan \\
WISP-1 & Wnt-1-induced secreted protein-1 \\
WT & wild type \\
\hline
\end{tabular}

\section{KARGER}

๑ 2008 S. Karger AG, Basel

Fax +4161306 1234

E-Mail karger@karger.ch

www.karger.com
Accessible online at: www.karger.com/cto
Dr. Marian F. Young, Molecular Biology of Bones and Teeth Unit, CSDB, DIR National Institute of Dental and Craniofacial Research, NIH Building 30, Room 225, 30 Convent Dr, MSC 4320 Bethesda, MD 20892-4320 (USA)

Tel. +1 301496 8860, Fax +1 301402 0824, E-Mail myoung@dir.nidcr.nih.gov 
BGN in vitro [Desnoyers et al., 2001]. Moreover, study of WISP-1 expression during development indicates that WISP-1 mRNA colocalizes with BGN during mineralization in vivo [French et al., 2004]. Considering the overlapping expression pattern and their ability to bind to each other, we hypothesized that BGN and WISP-1 might affect each other's capacity to control osteogenic cell function. Like the other CCN family members, WISP-1 has 4 distinct domains (fig. 2) [for review, see Leask and Abraham, 2006]. A naturally occurring variant of WISP-1 called WISP-1va lacks domain II which bears significant homology to von Willebrand factor CII and contains a putative TGF- $\beta$ /BMP-binding site [Tanaka et al., 2001]. Our data point to the possibility that WISP-1 and the variant WISP-1va can regulate BGN activity during osteogenesis.

\section{Materials and Methods}

WISP-1 expression was determined using immunofluorescence localization in human bone marrow stromal cells (hBMSCs) [Kuznetsov and Gehron Robey, 1996] or by Western blot analysis using protein extracts derived from cultured hBMSCs, mouse bone marrow stromal cells (mBMSCs) and from the long bones of 3-month-old wild-type (WT) mice. To detect WISP-1 in cells, an antibody designated LF-185 was raised against a peptide with the sequence RDTGAFDAVGEVEAWHRNC which corresponds to human WISP-1 isoform-1 amino acids 198-216 (accession No. NP-003873). A second antibody, designated LF-187, was raised using peptide with the sequence CRNPNDIFADLESYPDFEEIAN, corresponding to amino acid residues 346-367 located at the carboxy terminus of WISP-1. This latter sequence was conserved between mice and humans. The peptides used to make these antisera were first conjugated through the cysteine-to-maleimide-activated keyhole limpet hemocyanin (Pierce, Rockford, Ill., USA) and injected $(4 \times 1 \mathrm{mg}$ into multiple sites at approximately 2 -week intervals) into New Zealand White rabbits at an AAALAC-approved facility (Covance, Denver, Pa., USA). The serum antibody, LF-185, was affinity purified on a SulfoLink column (Pierce) onto which the same peptide had been covalently linked and then purified using conditions recommended by the manufacturer. LF-185 and LF-187 worked in direct ELISA on microtiter plates, immunocytochemistry and in Western blot (see below).

For immunofluorescence detection of WISP-1, hBMSCs were plated on glass coverslips, fixed, blocked in $10 \%$ goat serum $/ 0.1 \%$ Triton in PBS and then incubated with a 1:1,000 dilution of LF-185 followed by incubation with FITC-conjugated goat anti-rabbit $(1: 1,000)$ secondary antibody. Western blots were performed again using the LF-187 antibody with a horseradish peroxidaseconjugated secondary antibody following standard procedures.

The functions of BGN and WISP-1 were examined using both 'gain' and 'loss' of function approaches measuring proliferation and osteogenic capacity as our functional outputs. For gain of function, an adenovirus expressing BGN under the cytomegalovirus promoter was constructed using the full coding region of the mouse BGN cDNA (clone-3 in http://csdb.nidcr.nih.gov/csdb/ frame_reagents.htm) cloned into the shuttle vector, pACEF1. pLpA. hBMSCs were infected with the BGN-expressing adenovirus using $1 \times 10^{6}$ particles/well of a 96-well plate. As a control, an empty adenovirus containing no cDNA insert was used. In this part of the study, to test the effect of WISP-1, we used recombinant human full-length WISP-1 (rhWISP-1, $100 \mathrm{ng} / \mathrm{ml}$; Peprotech, Rocky Hill, N.J., USA). For loss of function, mBMSCs were taken from BGN-deficient mice [Xu et al., 1998] and cultured as described previously [Chen et al., 2002]. To analyze the effect of WISP-1 and its variant in the context of BGN depletion, DNA constructs encoding full-length human WISP-1 and another construct that lacks a von Willebrand factor CII domain (WISP-1va) were generated in PCDNA 3.1+ and then transfected into the mutant cells using the AMAXA nucleofector system and a protocol designed for mesenchymal stem cells.

Following treatment or transfection, cells were analyzed for effects upon proliferation and osteogenic commitment by measuring BrdU incorporation and alkaline phosphatase (AP) assays, respectively. For the proliferation assay, cells were incubated with $10 \mu \mathrm{M} \mathrm{BrdU}$ solution for $6 \mathrm{~h}$ before assay of BrdU incorporation with the chemiluminescent BrdU incorporation ELISA assay kit (Roche, Indianapolis, Ind., USA), using no BrdU incorporation, no antibody incubation and incubation of chemiluminescent reagent in empty wells as assay controls. The values obtained using these controls were subtracted from the experimental values to give the numbers shown in the $y$-axis of figure $3 a$. AP activity was measured using the colorimetric paranitrophenol phosphate assay (Sigma 104 kit; Sigma, Atlanta, Ga., USA) 5 days after transfection with or without the treatments described above. Briefly, cells were lysed with a solution of $20 \mathrm{mM}$ Tris, $0.5 \mathrm{mM} \mathrm{MgCl}_{2}$ and $0.1 \mathrm{mM} \mathrm{ZnCl}_{2}$, containing $0.1 \%$ Triton X-100. Ten microliters of cell lysate distributed to 96-well plates was incubated with $90 \mu \mathrm{l}$ of $1 \mathrm{mg} / \mathrm{ml}$ Sigma 104 paranitrophenol phosphate substrate solution in a buffer of $0.02 \mathrm{M} \mathrm{NaHCO}_{3}$ with $3 \mathrm{mM} \mathrm{MgCl}_{2}$ for $10 \mathrm{~min}$ at room temperature. The reaction was terminated using $0.5 \mathrm{M}$

Fig. 1. a Expression of WISP-1 in isolated hBMSC shown by immunofluorescence and confocal microscopy. Red staining represents positive immunostaining for WISP-1 using LF-185, while blue staining shows the nuclei imaged with DAPI. b Western blot of protein extracts obtained from hBMSCs (lane 1), mBMSCs (lane 2) and 3-month-old long mouse bones (lane 3) using LF-187. The size of WISP-1 appears to be larger in human cells and is relatively more abundant in mineralized tissues compared to cultured cells derived from those tissues.

Fig. 2. Domain structure of human WISP-1 (a) and WISP-1va (b). The $\mathrm{N}$ terminus is shown as a circle on the left side of the stick figure followed by a signal peptide (SP), the insulin growth factor-binding protein (IGFBP), von Willebrand factor type C (VWC), thrombospondin protein domain (TSP1) and a cysteine knot (CT). The gene accession numbers for human WISP-1 and WISP-1va splice variant are given in parentheses above each diagram. The numbers in parentheses above each exon indicate the number of nucleotides in that exon, while the numbers below the protein diagrams indicate the amino acid positions delineating the domain structures. 


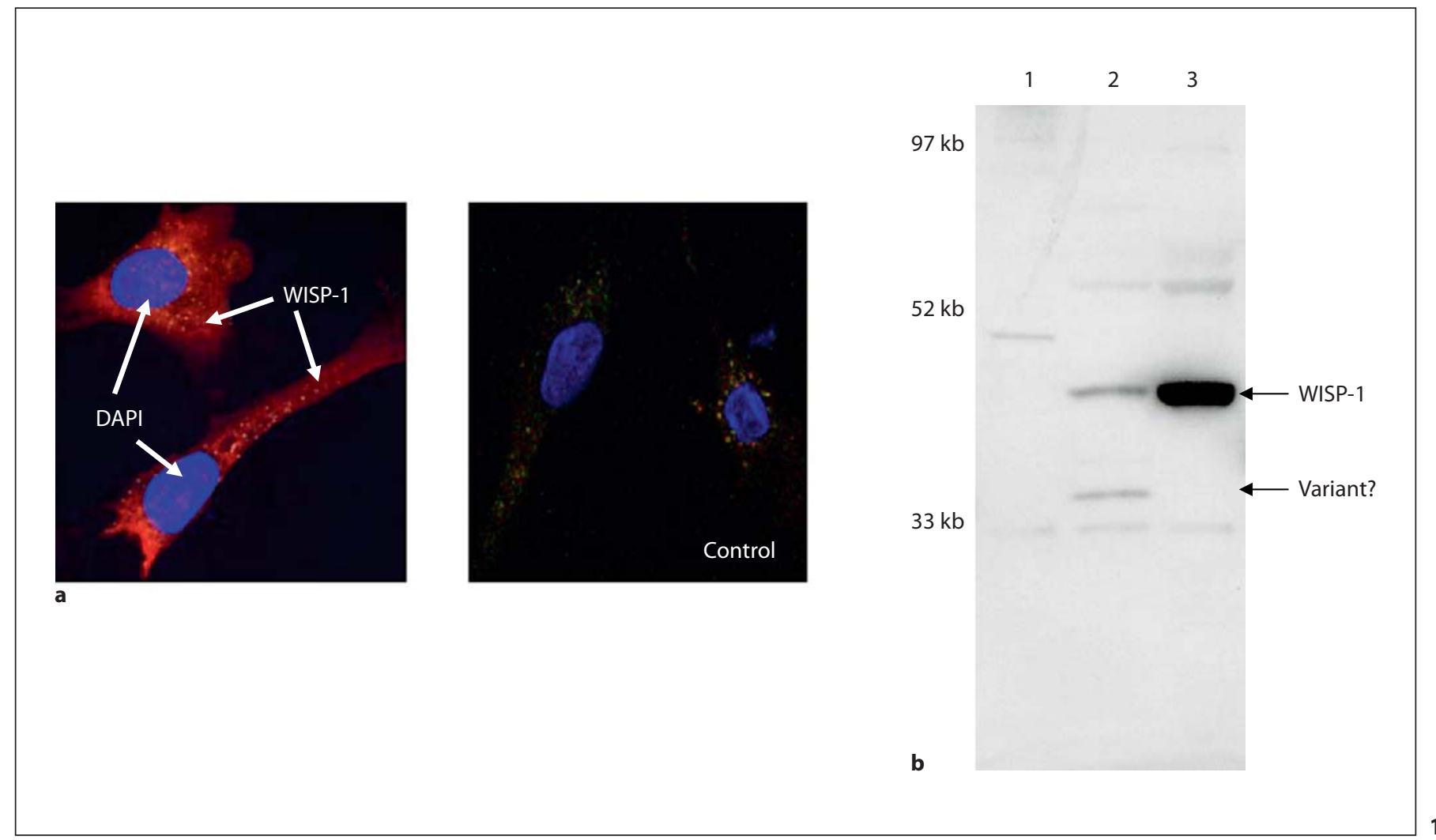

Human WISP-1 (AF100779)

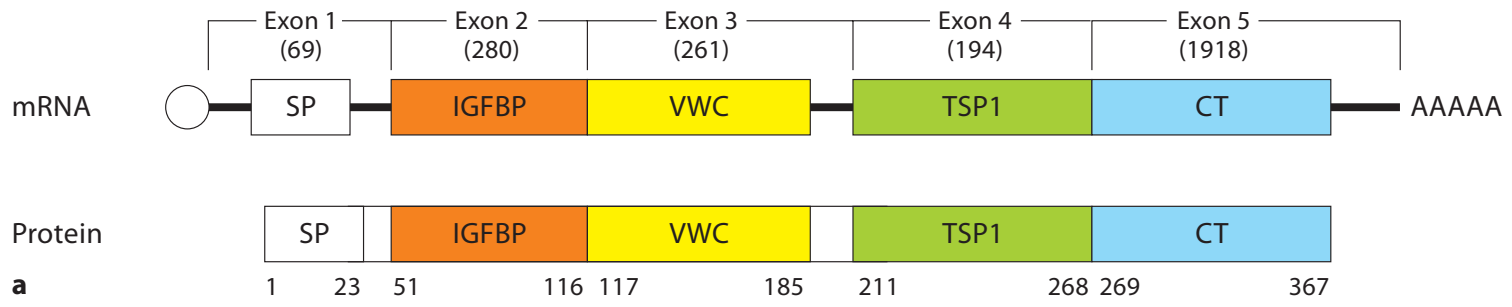

Human WISP-1 va splice variant (NM_003882)

\begin{tabular}{|c|c|c|c|c|}
\hline \multirow[b]{2}{*}{ mRNA } & xon & - Exon 2 & Exon 4 & Exon 5 \\
\hline & SP & IGFBP & TSP1 & $\mathrm{CT}$ \\
\hline Protein & SP & IGFBP & TSP1 & $\mathrm{CT}$ \\
\hline
\end{tabular}




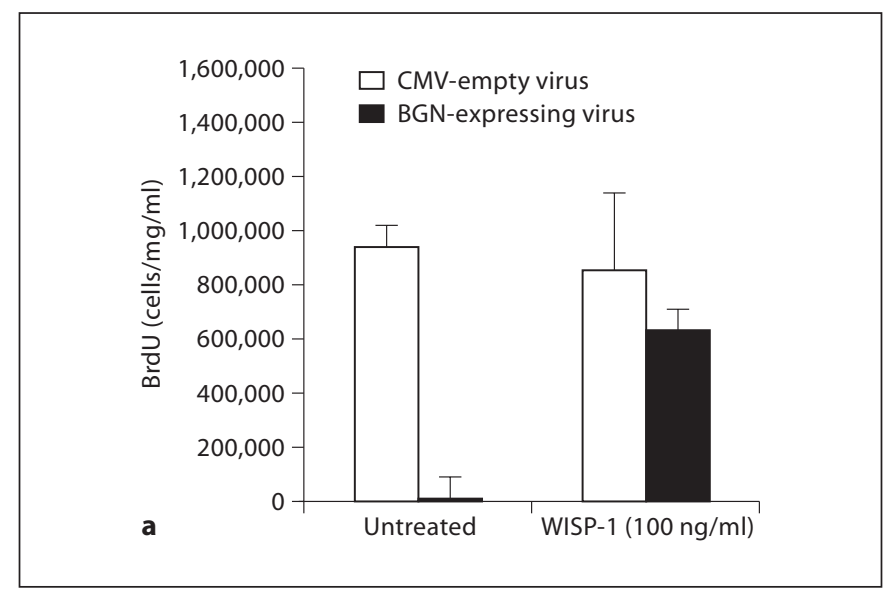

Fig. 3. a BrdU cell proliferation assay. hBMSCs were infected with $1 \times 10^{6}$ particles of adenovirus-expressing BGN per well of a $96-$ well plate. Twenty-four hours after the infection, some cells were treated with WISP-1 (100 ng/ml) for an additional $16 \mathrm{~h}$, following which cells were incubated with BrdU and incorporation assayed. $\mathrm{p}<0.016$ for cytomegalovirus-empty versus BGN virus mice. b AP assay of osteogenic differentiation. Bone marrow stromal

$\mathrm{NaOH}$ and the color development measured using a Victor $3^{\circledR}$ Wallac spectrophotometer plate reader (Perkin Elmer, Waltham, Mass., USA) at 405-nm absorbance. Total protein content of cell lysates was determined using the BCA protein assay kit described above and AP measurements were normalized to micrograms of protein per milliliter of sample.

\section{Results}

Using newly generated antibodies to WISP-1, we found that the protein was expressed in hBMSCs (fig. 1) and mBMSCs (fig. 1b, lane 2) cultured in vitro. However, its abundance was substantially less than that found in extracts of bone tissue in vivo (fig. $1 \mathrm{~b}$, lane 3 ). The major protein species of WISP-1 was different in human and mouse tissue with the human form being larger (fig. 1b). Minor smaller protein species were also apparent and may represent one or more of the variants previously described in the NCBI Entrez database. WISP-1 protein is composed of modules (fig. 2a) and there is a splice variant (WISP-1va) that lacks the von Willebrand factor type C domain (fig. 2b). We sought to determine the role of WISP-1 and its splice variant in modulating BGN functions in bone marrow stromal cells.

When adenovirus-expressing BGN was transduced into hBMSCs, it had inhibitory effects on proliferation (fig. 3a) judged by BrdU incorporation assays if compared

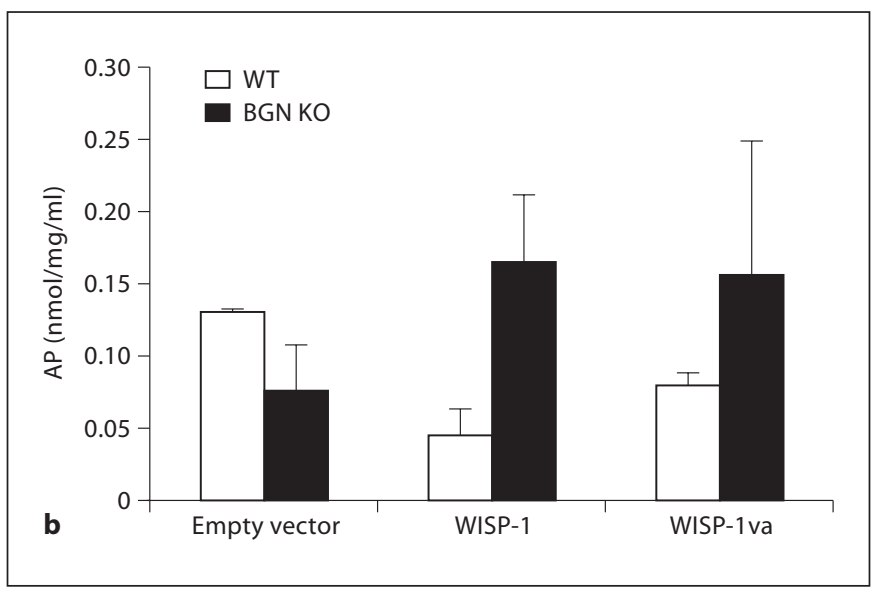

cells were isolated from normal and BGN-deficient mice and transfected with vector encoding full-length human WISP-1 or WISP-1va empty vector (PCDNA 3.1+) using an AMAXA nucleofector system. AP was measured 5 days after transfection. $\mathrm{p}<0.05$ for WT mice treated with WISP-1 versus BGN KO mice treated with WISP-1.

to infection using the same concentration of an empty control adenovirus. The addition of rhWISP-1 alleviated BGN-inhibited proliferation, to the same extent as by TGF- $\beta$, another protein known to bind to BGN (data not shown).

Previous studies from our laboratory have found that BGN-deficient osteoblasts have a decreased osteogenic potential, judged by their reduced AP activity, compared to WT controls [Chen et al., 2004]. To test the role of WISP-1 and WISP-1va on AP activity, WT and BGN-deficient mBMSCs were transfected with both WISP-1 and WISP-1va constructs. After 5 days, both WISP-1 and WISP-1va 'rescued' mutant BGN-deficient cells, and increased AP activity was higher than that observed in the WT cells (fig. 3b). The differences observed between WT and BGN cells in response to WISP-1 may have been influenced by the fact that WISP-1 decreased in the AP in cells transfected with control empty vector.

\section{Discussion}

For many years, our laboratory has been investigating the role of the extracellular matrix proteoglycan BGN in regulating mineralized tissue formation. Early studies showed that mice deficient in BGN had age-related osteopenia due to a defect in osteoblast differentiation and 
maturation [Chen et al., 2004]. In addition to this, our data revealed a role for BGN in modulating BMP accessibility and responsiveness; however, the role of additional associating factors that could contribute to this modulation remains elusive.

In an attempt to identify binding partners for WISP-1, Desnoyers et al. [2001] found that one of the major proteins that binds to WISP-1 is BGN. WISP- 1 is a CCN family member that is upregulated in many types of tumor tissue [Leask and Abraham, 2006] and in response to the Wnt/ $\beta$-catenin signaling pathway [Pennica et al., 1998]. Previous studies showed that WISP-1 is expressed in osteogenic cells both during development and at sites of new bone formation during fracture healing [French et al., 2004]. Our work confirmed that WISP-1 is produced by human osteogenic cells and that it accumulates in the matrix of bone in vivo.

Considering the fact that BGN and WISP-1 colocalize to mineralizing tissue and that they physically bind to each other, we reasoned that they might influence each other's activities in skeletal tissues. Thus, the aim of our study was to test whether BGN and WISP-1 work together to modulate osteogenesis. Our data point to the possibility that WISP-1 counteracts the antiproliferative action of BGN in osteogenic precursors. In addition, WISP1 and its alternatively spliced variant appear to rescue the diminished osteogenic differentiation caused by the absence of BGN. It is further interesting to note that BGN- deficient osteogenic cells have less expression of WISP-1 and that this can be rescued by addition of exogenous BGN (data not shown). While these data provide a foundation to link BGN action with WISP-1, it is still not clear if a physical interaction is needed for these effects. It would be interesting to further determine whether there are any other factors that might work in concert with WISP-1 and BGN. Likely candidates in this regard would be one or more of the TGF- $\beta /$ BMP family members. TGF- $\beta$ and BMP have previously been shown to bind to both SLRPs and CCN family members. Future experiments will be designed to test whether TGF- $\beta$ affects either BGN or WISP-1 functions and, further, to determine the precise sequence of events that unfolds with this triad in bone and tooth formation during development or after repair of damaged tissue.

\section{Acknowledgements}

Kind thanks to Dr. S. Kesavapany (Department of Biochemistry, Yong Loo Lin School of Medicine, Singapore) for help with confocal microscopy and Dr. S. Tanaka (Department of Biochemistry and Molecular Dentistry, Okayama University Graduate School of Medicine, Dentistry and Pharmaceutical Sciences, Okayama, Japan) for WISP-1va clones. This work was supported by the Division of Intramural Research, National Institute of Dental and Craniofacial Research of the Intramural Research Program, National Institutes of Health, Department of Health and Human Services.

\section{References}

Boskey, A.L., L. Spevak, S.B. Doty, L. Rosenberg (1997) Effects of bone CS-proteoglycans, DS-decorin, and DS-biglycan on hydroxyapatite formation in a gelatin gel. Calcif Tissue Int 61: 298-305.

Chen, X.D., L.W. Fisher, P.G. Robey, M.F. Young (2004) The small leucine-rich proteoglycan biglycan modulates BMP-4-induced osteoblast differentiation. FASEB J 18: 948-958.

Chen, X.D., S. Shi, T. Xu, P.G. Robey, M.F. Young (2002) Age-related osteoporosis in biglycandeficient mice is related to defects in bone marrow stromal cells. J Bone Miner Res 17: 331-340.

Desnoyers, L., D. Arnott, D. Pennica (2001) WISP-1 binds to decorin and biglycan. J Biol Chem 276: 47599-47607.

Fisher, L.W., J.D. Termine, S.W. Dejter Jr, S.W. Whitson, M. Yanagishita, J.H. Kimura, V.C. Hascall, H.K. Kleinman, J.R. Hassell, B. Nilsson (1983) Proteoglycans of developing bone. J Biol Chem 258: 6588-6594.
French, D.M., R.J. Kaul, A.L. D’Souza, C.W. Crowley, M. Bao, G.D. Frantz, E.H. Filvaroff, L. Desnoyers (2004) WISP-1 is an osteoblastic regulator expressed during skeletal development and fracture repair. Am J Pathol 165: 855-867.

Kuznetsov, S., P. Gehron Robey (1996) Species differences in growth requirements for bone marrow stromal fibroblast colony formation in vitro. Calcif Tissue Int 59: 265-270.

Leask, A., D.J. Abraham (2006) All in the CCN family: essential matricellular signaling modulators emerge from the bunker. J Cell Sci 119: 4803-4810.

- Parisuthiman, D., Y. Mochida, W.R. Duarte, M. Yamauchi (2005) Biglycan modulates osteoblast differentiation and matrix mineralization. J Bone Miner Res 20: 1878-1886.

Pennica, D., T.A. Swanson, J.W. Welsh, M.A. Roy, D.A. Lawrence, J. Lee, J. Brush, L.A. Taneyhill, B. Deuel, M. Lew, C. Watanabe, R.L. Cohen, M.F. Melhem, G.G. Finley, P.
Quirke, A.D. Goddard, K.J. Hillan, A.L. Gurney, D. Botstein, A.J. Levine (1998) WISP genes are members of the connective tissue growth factor family that are up-regulated in Wnt-1-transformed cells and aberrantly expressed in human colon tumors. Proc Natl Acad Sci USA 95: 14717-14722.

Tanaka, S., K. Sugimachi, H. Saeki, J. Kinoshita, T. Ohga, M. Shimada, Y. Maehara, K. Sugimachi (2001) A novel variant of WISP1 lacking a von Willebrand type $\mathrm{C}$ module overexpressed in scirrhous gastric carcinoma. Oncogene 20: 5525-5532.

Xu, T., P. Bianco, L.W. Fisher, G. Longenecker, E. Smith, S. Goldstein, J. Bonadio, A. Boskey, A.M. Heegaard, B. Sommer, K. Satomura, P. Dominguez, C. Zhao, A.B. Kulkarni, P.G. Robey, M.F. Young (1998) Targeted disruption of the biglycan gene leads to an osteoporosis-like phenotype in mice. Nat Genet 20: $78-82$. 